\title{
The development of an ESEM based counting method for fine dust particles and a philosophy behind the background of particle adsorption on leaves
}

\author{
M. Ottelé ${ }^{1}$, W. J. N. Ursem ${ }^{2}$, A. L. A. Fraaij ${ }^{1}$ \\ \& H. D. van Bohemen ${ }^{1}$ \\ ${ }^{1}$ Faculty of Civil Engineering and Geosciences, \\ Delft University of Technology, The Netherlands \\ ${ }^{2}$ Department of Biotechnology, Faculty of Applied Sciences, \\ Delft University of Technology, The Netherlands
}

\begin{abstract}
The multi scale benefits of urban greenery (green façades and green roofs) have attracted more and more interest of recent research work. The multi scale benefits of vegetation vary from; mitigation of the urban heat island effect, stimulation of the ecological value and biodiversity, aesthetical reasons and for example air pollution reduction. Air pollution control is at the moment mainly focussed on the reduction of fine particle concentrations. Particulate air pollution is damaging for the human health, it causes cardiovascular and lung diseases. Especially dust particles smaller than 2.5 micrometers are of great interest because they can be deeply inhaled into the respiratory system. To determine the effect of leaves on particle adsorption, micrographs are taken of ivy (Hedera helix) leaves using an Environmental Scanning Electron Microscope (ESEM). The examined leaves are exposed to a simulated rainfall in order to determine a method for particle counting on leaves and to determine the self cleaning effect of adsorbed particles on ivy leaves. The self cleaning effect is considered to be an important factor in the effectiveness of particle adsorption by leaves and the potential for resuspension of particles. Particles on pre- and post-rain leaves were counted via the ESEM micrographs using an image analyzer. Results showed that there is no significant effect on particle loss due to rain in the performed experiment. Our findings suggest that a strong Van der Waals bonding between
\end{abstract}


particle and leaf surface plays an important role in the retaining process of fine particles on the leaf surface.

Keywords: green façade, fine particle accumulation, environmental scanning electron microscope, simulated rainfall, Hedera helix.

\section{Introduction}

Green façade designs offer numerous economic, social and environmental benefits such as greenhouse gas emission reduction, adaptation to climate change, air quality improvements, habitat provision and improved aesthetics. Also sound reductions are possible by the use of vegetation [1]. Despite these benefits, a widespread market penetration of greening technologies over the world remains still in its infancy.

Greening the façades of urban buildings or infrastructural projects (i.e. sound barriers, tunnel alignments, etc.) using climbing plants or living wall systems (LWS) modifies the interaction of the building system with the surrounding atmosphere. For example: plants have the ability to dissipate absorbed solar radiation into sensible and latent heat [2]. This is often related to the urban heat island effect, but it has also an effect on the vertical air velocity [3] and is thus (indirectly) related to particle deposition processes. With other words, a specific microclimate will be created around a green building envelope. This microclimate could not only improve the outdoor or indoor climate but will also have an effect on the distribution and accumulation of particles inside the street canyon due to the filtering capacity of climbing plants [4]. The aim of this paper is to classify and to provide a first step in a comprehensive study on the potential use of green façades to improve the local air quality in urban environments.

In the presented research, adsorption and removal of particulate matter by vegetation are discussed because of the associated increased morbidity and mortality aspects of inhalation fine atmospheric particles by humans. Especially finer particles (those with an aerodynamic diameter of $<10 \mu \mathrm{m}\left(\mathrm{PM}_{10}\right)$, pose a long-term threat to the human health, mainly to the human respiratory functions [5]. In general, the smaller the particles, the deeper they penetrate into the respiratory system were it is taken up into the blood. In this way, respiratory and/or cardiovascular disease arise [5]. Research done by Pope et al. [6] in the United States on the life expectancy of humans and the concentration of fine particulate air pollution, shows that there is an increase in life expectancy when there is a decrease of $10 \mu \mathrm{g}$ per cubic meter in the concentration of fine particles in the ambient air.

Literature review shows the importance of altering the amount of fine particles in the air to improve human health. Although this information, less research has been done on the potential impact of lowering the amount of fine particles by vertical greened surfaces and on counting the amount of adsorbed fine particles by leaves.

Past research methods focussed on particle mass levels through examine the effluent of washing urban tree leaves [7-10]. In assessing the potential benefit of fine particle adsorption by green façades this paper examines if there is an 
influence of rainfall simulation on particle retention by vegetation. Besides that the paper intended to classify the amount of particles by a counting method based on Environmental Scanning Electron Microscope (ESEM) images. The method of using micrographic images in combination with an image analyzer (Image $J$ ) enables to study and identify particle size, origin and amount directly on leaves. Surfaces of vegetation are recognized as a terrestrial sink for atmospheric particulate matter, consisting of particles which are highly variable with respect to origin, chemical and physical properties, elemental composition, and potential biological and environmental impact. Vertical greened surfaces may be especially efficient filters of airborne particles [11] because of their high surface to volume ratio of foliage, abundant petioles and twigs, and due to hairy, wax structure or rough leaf surfaces and shapes. To estimate the filtering effects of façade greening, it is necessary to study the relationships between the retention of particles on the leaf surfaces and the local pollutant concentration. Also the resuspension of already adsorbed particles is an important parameter in the purification process. Processes which have to be taken into account for resuspension of particles are among other things: wind, rainfall and falling of leaves (figure 1).

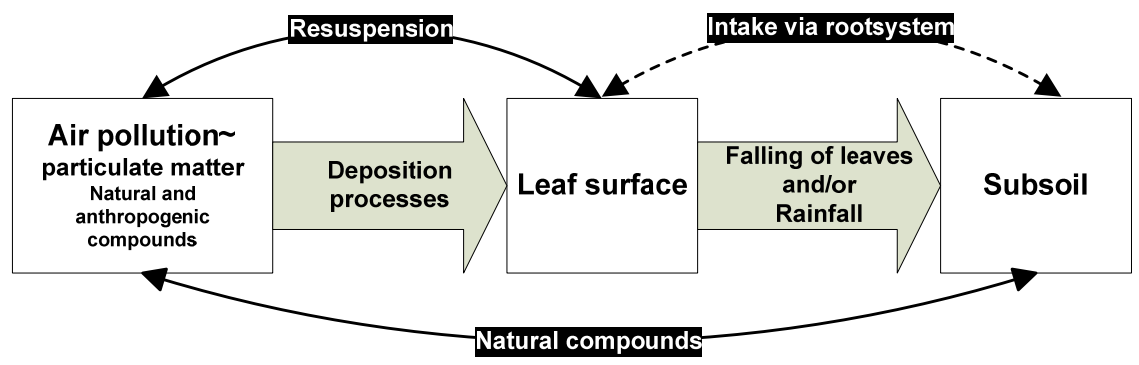

Figure 1: Conceptual model particle circulation.

A literature survey undertaken by the authors on the different mechanisms between particles and leaves shows that resuspension of particles is commonly stated but hardly investigated. Once fine particles are adsorbed by the leaf surface it is important to know if they still form a danger for human health as discussed earlier. Two main forces act on small, moving airborne particles: one is the force of gravity and the other is the viscous force exerted by the air through which the particles are moving [12]. If the particles are electrically charged they will also be subject to electrostatic forces, which may alter the dispersal and deposition patterns of charged particles compared with uncharged ones [12]. Some particles may be adsorbed into the tree but most are retained on the plant surface [11]. Once particles are adsorbed to the leaf surface it is important to know the pathway that will bring these particles to the subsoil. Also the particle sizes involved in this process are of interested. In this paper a simulated rainfall experiment was carried out on Hedera helix leaves (common ivy) to get more insight into the percentage of (fine) particles that can be washed 
off or that will remain on the leave surface with the potential to end up (resuspended) in the air again. According to Nowak et al. [13] the resuspension of particles can be up to $50 \%$ of the adsorbed particles. The objective of this study aimed in defining the reduction of air pollution and stimulating the ecological performance of vertical green (green façades, sound barriers, etc.) with plants. The interaction or filtering effect between (vertical used) vegetation and air pollution (particulate matter) is not well studied yet. Especially the effect of rainfall on particle retention and resuspension is unclear. The approach in this research is not to measure the mass of particles collected on the leaves but the amount of adsorbed particles. Measuring the mass of particles instead of the size and amount of particles ignores the risk for human health with respect to hearth and lung diseases. Since particle size is correlated with lung diseases (it is important to know how many of those fine or ultra fine particles are adhered on the leaf surface. Counting particles on a specific leaf area seems therefore more suitable for this experiment.

\section{Materials and methods}

Leaves of common ivy (Hedera Helix) were chosen for this experiment. The leaves were collected from a vertical greened fence near Steenbergen (The Netherlands) in the beginning of October 2008, after a period of 6 days without rain. Eleven adult ivy leaves and only entire green leaves were taken randomly from the outside ivy foliage of the greened fence. The collected leaves were sealed and labelled separately in plastic containers to exclude the possibility of contamination after sampling. The sealing procedure was done in a way to keep the leaf surface untouched until the examination in the Microlab of Delft University of Technology. To distinguish the difference between the amount of particulate matter before and after rainfall on the leaves (only the upper side of the leaf is examined in this research), micrographs where taken with an Environmental Scanning Electron Microscope (Philips XL30 ESEM with a tungsten filament) at different magnifications namely 100x, 500x and 5000x. An experimental procure was addressed in order to make micrographs before and after rainfall simulation on the same leaf and on the same spot, valid for each magnification. The micrographs are always taken in the middle of the leaf lefthanded near the central nerve (figure 2, right photograph). When a spot is found, the spot is fixed in the middle of the view and the micrographs are taken at different magnifications. After this session the leaf is exposed to a simulated rainfall. Therefore the leaves were placed in a tripod on a bench in which all rainfall events were performed. Rainfall was simulated with a pressurized system utilizing a rain nozzle designed to project a downward spray. The nozzle was placed about $60 \mathrm{~cm}$ above the leave(s) and a uniform spray was attained to simulate the rainfall. The leaves were subjected to a simulated rainfall event of 15 minutes at a rainfall rate of $80 \mathrm{mmh}^{-1}$ with normal tap water $(\mathrm{pH} 8.0)$ measured with a funnel with a diameter of $13 \mathrm{~cm}$.

A relative high rainfall rate was chosen for this experiment to make sure that the leaf was fully wetted. After the simulated rainfall event, the leaf was placed 
again in the ESEM chamber with the positioning holder (figure 2, left photograph). Micrographs were taken at the same spot as before the rain simulation. This procedure was repeated for each of the collected leaves. In this system the computer is responsible for beam control, image analysis, data processing and data storage. For image analysis, the backscattered electron signal is used to create a binary image of the leaf surface with the adhered particles.

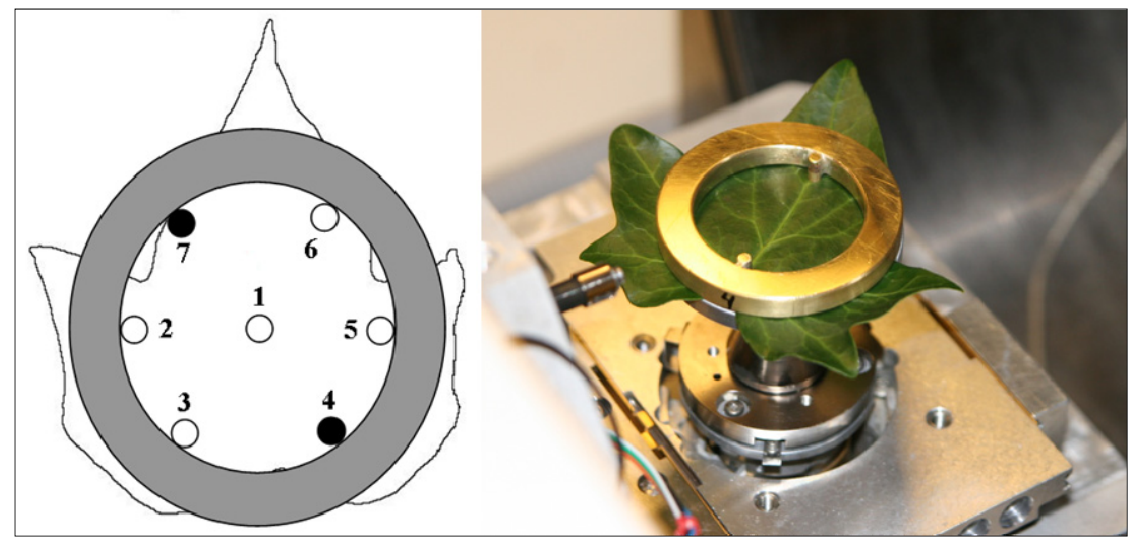

Figure 2: $\quad$ Sampling procedure using ESEM analysis.

After collecting microphotographs the particle counting was done automatically with an image analyzer software package called Image J. Particle analysis requires that the image is a "binary" image (i.e. black or white). The biggest issue is to distinguish the particles from the background (threshold). See figure 3 and figure 4 to go from raw to threshold. The automatic threshold function used by Image $J$ was applied, in some cases manual correction of the automatic threshold value was needed. Further information about the program can be found on [14]. Particles that are slightly overlapping in a threshold image must be separated; this was done with the watershed function in Image J. Once the particles have been successfully threshold and watershed, they can be analyzed to obtain information regarding particle size and numbers. In the analysis no boundary to the circularity value was given (i.e. a value of 1.0 indicates a perfect circle), which means that all various shapes of particles were count. Per magnification (100x, 500x, respectively 2500x) the different adsorbed particles were count, also weight factors, respectively 1, 25 and 2500 times, were used to compensate for the loss of counting area (zoom effect). In addition, the cross sectional diameters of each of the particles were calculated by assuming that a calculated area belongs to a certain aerodynamic diameter. The experiment and procedure for counting was repeated and carried out for each individually examined leaf.

The presented counting procedure was done by second year's students from the Faculty of Civil Engineering of Delft University of Technology and the students are instructed how to analyze the micrographs. Each leaf was measured 

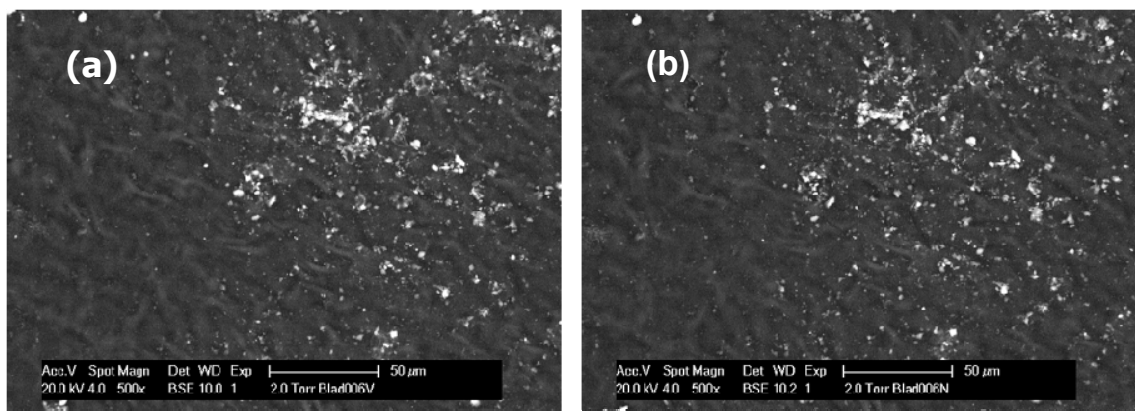

Figure 3: (a) ESEM micrograph before rainfall, and (b) after rainfall.
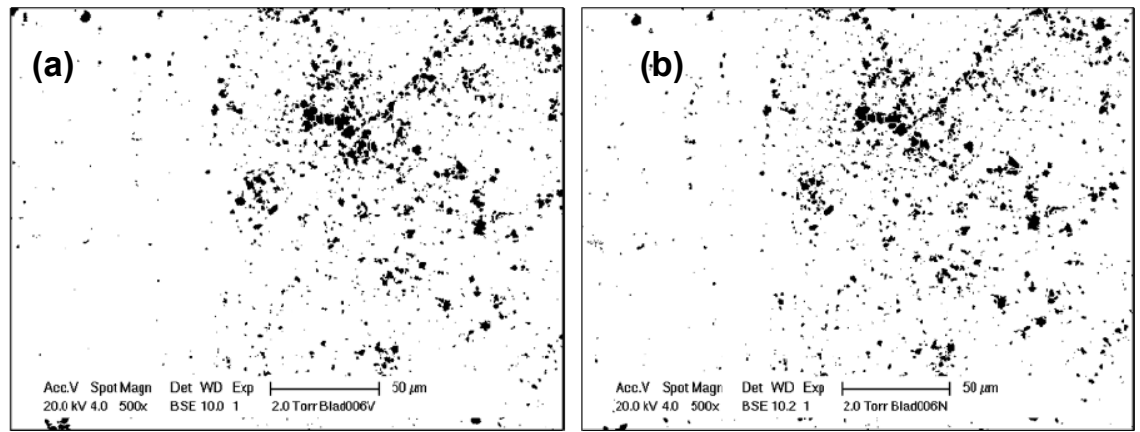

Figure 4: $\quad$ (a) Threshold micrograph before and (b) after rainfall.

by a new group of students, a group exists out of six students and each individual student in a group analyzes the same photo series before and after simulated rainfall. The groups of students were used to examine if there is an influence on the sensitivity of the used image analyzing procedure. The total of used specimens (different leaves) and thus also the number of groups for this experiment was eleven (each leaf is thus counted by six different persons). The collected data (response variable) from the counting procedure was evaluated by an replicated random block design (ANOVA) to test an eventual influence of the students (independent variable) on the counting procedure and the effect of simulated rainfall (independent variable) on particle loss Each collected data set was filtered (the six outcomes of the counting procedure for each leaf before and after rainfall) by testing for outliers. This filtering procedure was done on the basis of Chauvenet's criteria assuming that each individual data set follows the normal distribution. The rejected outliers were considered as a "malfunctioning" of the students. The statistical analysis was performed by SPSS 16.0 software package. The level of significance used in the analysis and throughout the paper is alpha $5 \%$. The outcome of this analysis will give more insight into the relevance and interactions between the retention of particles on leaf surfaces and the influence of rainfall on the retention. Also the sensitivity of the counting procedure (with relation to the need of qualified personnel) can be examined. 
The statistical outcome enables also $\mathrm{H}_{\varnothing}$ hypothesis checking. In this study, we hypothesized that rain changes the amount of adsorbed particulate matter on ivy leaves and that students will not have an effect on the outcome of counting particles.

\section{Results}

\subsection{Outcome of counting particles before and after simulated rainfall}

The result of counting particles on a specific leaf area $\left(950 \times 1275 \mu \mathrm{m}^{2}\right)$ results in a particle distribution (figure 5) for the upper side of the leaf. Particle sizes $\geq 10 \mu \mathrm{m}$ appeared to be rather rare compared to particles sizes $\leq 10 \mu \mathrm{m}$.

Table 1: Outcome of counting fine particles based on ESEM images; before and after simulated rainfall.

\begin{tabular}{|c|c|c|c|}
\hline \multirow{2}{*}{$\begin{array}{l}\text { Blocks } \\
(n=11)\end{array}$} & \multicolumn{3}{|c|}{ Treatment } \\
\hline & $\begin{array}{c}\text { before rain }(x) \\
\text { average out of six students } \\
(n=6)\end{array}$ & $\begin{array}{c}\text { after rain }(y) \\
\text { average out of six students } \\
(n=6)\end{array}$ & $\begin{array}{c}\text { difference } \\
(x-y)\end{array}$ \\
\hline leaf 1 & 96952 & 81396 & 15556 \\
\hline leaf 2 & 78277 & 64609 & 13668 \\
\hline leaf 3 & 34648 & 27410 & 7238 \\
\hline leaf 4 & 33376 & 28296 & 5080 \\
\hline leaf 5 & 86644 & 87235 & -591 \\
\hline leaf 6 & 130134 & 51011 & 79123 \\
\hline leaf 7 & 29285 & 21016 & 8269 \\
\hline leaf 8 & 75499 & 52146 & 23353 \\
\hline leaf 9 & 199813 & 160142 & 39670 \\
\hline leaf 10 & 185010 & 164838 & 20172 \\
\hline leaf 11 & 48413 & 144531 & -96118 \\
\hline
\end{tabular}

Particles larger than $10 \mu \mathrm{m}$ will thus not be studied in this paper. Almost all of the peaks were found in the range up to $2.5 \mu \mathrm{m}$. The smallest particles that are found with the applied measurement technique are in the range of $0.2 \mu \mathrm{m}$. Rainfall did not have a significant effect on the number of particles retained on the leaves. The number of particles (table 1) before the rainfall ranged from \pm 29000 to \pm 199000 particles per $\mu \mathrm{m}^{2}$ and was not significantly different from the number of particles (respectively \pm 21000 and 160000 particles per $\mu \mathrm{m}^{2}$ ) retained on the leaves after the simulated rainfall event (figure 5). For leaf 5 and 11 a negative value was found for the amount of particles after the measurement. This means more particles entered the counting area after the simulated rainfall event. 


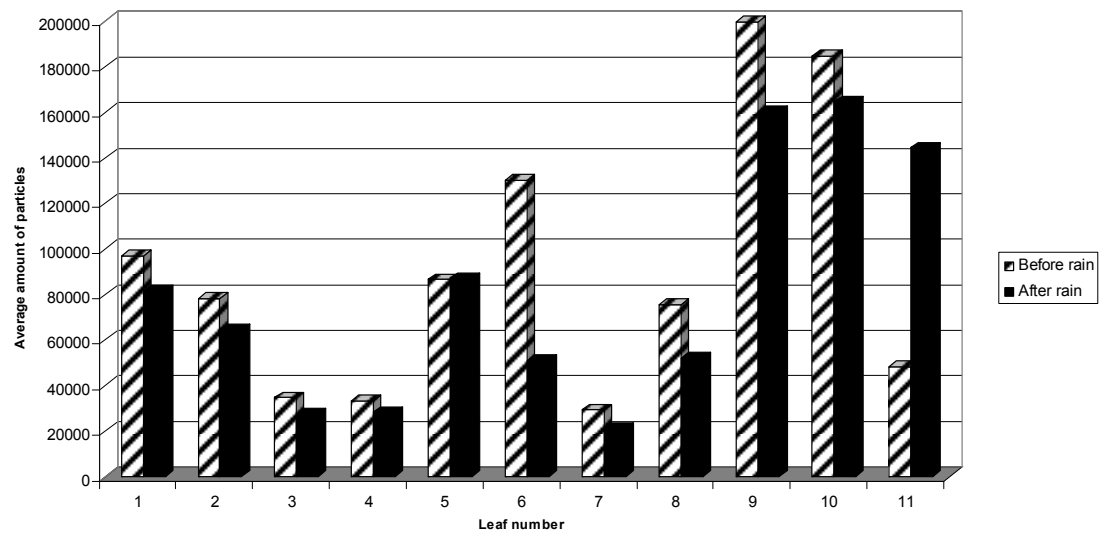

Figure 5: Average amount of particles found on Hedera helix leaves before and after simulated rainfall.

\subsection{Evaluation of statistical analysis}

Throughout this paper differences between samples are indicated to be significant if they were significant at $\alpha<0.05$ based on the results of the paired $t$ test (table 2). The F-test is done on each individual counting procedure. The outcome of the statistical analysis identified that in the presented study no significant differences were found between the treatments. The null hypothesis that rain may not have an effect $(\mathrm{P}=0.186)$ is thus rejected. However the hypothesis that students $(\mathrm{P}=0.000)$ will not influence the outcome of particle counting is accepted.

\section{Discussion}

In this paper the effect of rain on the cleaning effect of leaves has been studied. Eventual cleaning effect of leaves by rain will lower the chance that particles will be affected by wind loads (resuspension). Rainfall may influence thus (in) directly the capacity and efficiency of the leaf on particle reduction in the ambient air. Simulated rainfall shows that the expectations with respect to particle wash off and the self cleaning effect of Hedera helix leaves are far away from ideal. For leaf 5 and 11 consequently we found more particles in the counting area after the simulated rainfall. This means that particles are moved from the area around the examined spot into the counting area. With other words particles are not always fixed on the leaf surface which can be explained by figure 6 , were we see an accumulation of particles especially near the edge or tip of the leaf. The photograph also suggests that there is a particle transfer over the leaf surface. The overall result however of the experiment shows that the simulated rainfall has a minor effect on the self cleaning effect of Hedera helix leaves on particulate matter. This leads us to the next question whether particles retained on the leaf surface after the rainfall have the potential to end up 
(resuspended) into the air again due to wind turbulences. The question that can be raised concerning the outcome of this experiment is how likely this resuspension of particles is after a rainfall? Additional wind experiments concerning particle loss are necessary to detect the effect of air turbulence in regard to this statement.

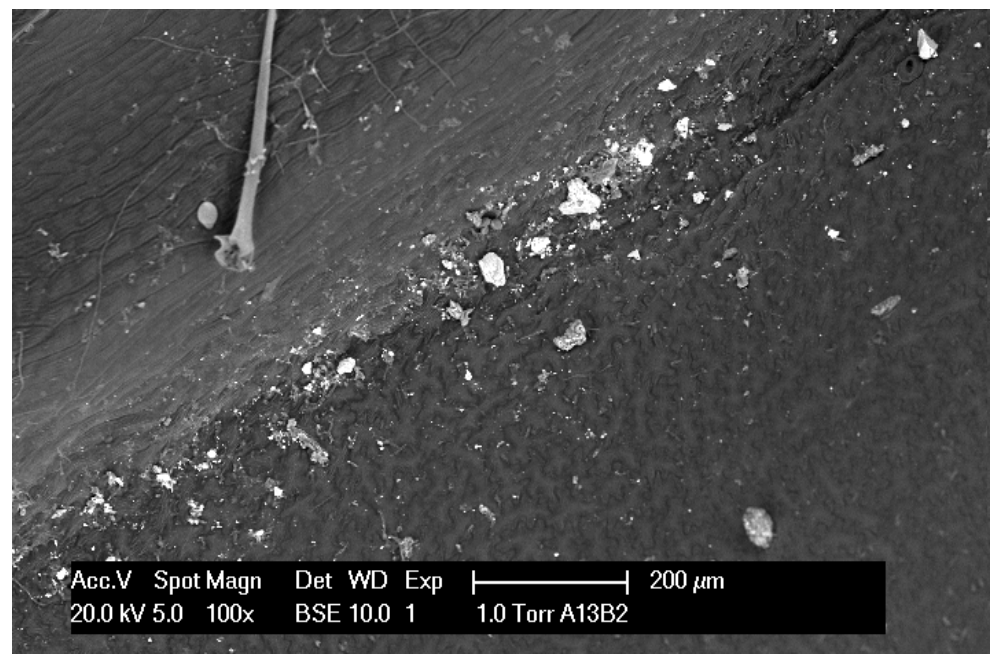

Figure 6: Accumulation and clogging of particulate matter on the edge of a leaf.

Most of the particles are still present on the leaf surface after the heavy simulated rainfall, so the particles are really well embedded on the leaf surface. Is there an explanation for the minor particle loss due to the effect of rainfall and that the particles are well embedded? Is there a role for the leave composition (wax layer, hairy, rough surface, etc.), the leaf boundary layer or electrostatic behaviour of particles and leaf surface? The wax layer can be of importance to stick the particles, but is most unlikely to be able to hold on after the rain experiment of a simulated rainfall event of 15 minutes at a rainfall rate of 80 $\mathrm{mmh}^{-1}$ with normal tap water $(\mathrm{pH} 8.0)$. Wax structures consist of saturated verylong-chain fatty acids (commonly $\mathrm{C}_{20}$ to $\mathrm{C}_{34}$ ) and in surface structure not equipped to hold fine dust particles [15-17]. A well understood surface wax structure of the Lotus, Nelumbo nucifera, is even been used for its properties of self cleaning effect, due to the laminar special wax structure [18]. This also explains the observed lacking of large fine dust particles of $10 \mu \mathrm{m}$ or more on the leaf surface at all. Besides the difficulty of fixation, all large particles of $10 \mu \mathrm{m}$ or more are washed away at a high relative humidity together with low temperatures, which could easily form a dew point effect on the leaf surface, or in cases of precipitation, or in situations of a dry environment due to wind turbulence. The hairs are lacking on common ivy, so this factor is of no importance concerning the fine dust particle adhesion. The leaf boundary layer is 
extremely related to the wax structure of the surface of the epidermal cells. As mentioned before, the leaf boundary layer and the fixation of fine dust particles of $10 \mu \mathrm{m}$ or more can been seen in the same way as the given structural analysis of wax surfaces. In conclusion, it is very difficult to hold these large fine dust particles of $10 \mu \mathrm{m}$ or more on the leaf surface of common ivy based on the leaf composition (wax layer, hairy, rough surface, etc.) or on the leaf boundary layer. If a boundary layer could be part of the adhesion factor after the mentioned rainfall, then it can only remain the adhesion properties of fine dust particles when there is a strong bonding of fixation. The only possible strong bonding on the molecular level which resists the test of a 15 minutes rainfall with a rate of $80 \mathrm{mmh}^{-1}$ will be a Van der Waals bonding. A Van der Waals bonding can only be induced due to a transition of another extra energy source, like an electrical charged fine dust particle.

The main sources of fine dust particles of $10 \mu \mathrm{m}$ or more are known from sand-, clay- or biological origin. In cases of smaller distributions, particles of less than $10 \mu \mathrm{m}$, the main origin is from gas exhaust of combustion and engines. The result of the findings in this research on common ivy shows clearly a dominant persistence of particulate matter smaller than $10 \mu \mathrm{m}$ in diameter. The samples of common ivy mentioned in this research are all exposed to the influence of traffic. Fine dust particles of combustion and engines consistently exhibits in the soot mode of 20 till $200 \mathrm{~nm}$ size with a substantial fraction of 40 till 60 percent of charged particles [19]. This means that more then half of all fine dust particles of combustion or engine origin are charged and these particles will be neutralized as soon as it touches the leaf surface. The common ivy is connected with its root system to the soil, so per definition charged in the same manner as the soil it self or in other words the electrical charge can be seen as grounded [20,21].

In short, the particulate matter either gains electrons in cases of positive charged fine dust particles or lose electrons in cases of negative charged fine dust particles. Because no electrical charged energy can get away in any other form, as soon as a fine dust particle touches the leaf surface of the common ivy, the electrical charge will be converted into a strong Van der Waals bonding. A Van der Waals bonding is known in physics to be an extreme strong fixation bonding, so this explains and underlines the positioned particulate matter on the leaves of common ivy after the 15 minutes at a rainfall rate of $80 \mathrm{mmh}^{-1}$.

\section{Conclusions}

Since the research was focused on the effect of rainfall on particle retention on the leaves of common ivy (Hedera helix) we can conclude that the cleaning effect of rainfall for the fine and ultra fine particles is very low. However, the observed phenomenon of the remaining of the particulate matter on the leaf surface brought us to basis principle of physics of a Van der Waals bonding as the only possible explanation of fixating after 15 minutes at a rainfall rate of 80 $\mathrm{mmh}^{-1}$. The electrical charged particles of gas exhaust of a combustion or engine source can be considered as the most important factor of the remaining of the particulate matter of less than $10 \mu \mathrm{m}$ on the leaf surface of common ivy of our 
research. The fine dust particles of $10 \mu \mathrm{m}$ or more have not been observed in this study on the leaf surface of all sampled common ivy. The adhesion of these relative large fine dust particles is possible washed or blown away as mentioned and discussed in this article.

\section{Acknowledgements}

The Microlab of Delft University of Technology, Faculty of Civil Engineering and Geosciences is acknowledged for the permission to make use of the necessary research facilities. The authors would also like to thank the second year students of the year 2008 for collecting the data (analyzing micrographs) needed for this experiment. We also thank Mr. A. Thijssen for his technical support, making the ESEM micrographs and data processing.

\section{References}

[1] Pal, A.K., Kumar, V. and Saxena, N.C., Noise attenuation by green belts, Journal of Sound and Vibration, 234-1, 149-165 (2000).

[2] Stec, W.J., Van Paassen, A.H.A. and Maziarz, A. Modelling the double skin façade with plants, Energy and Buildings, 37, 419-427 (2005).

[3] Minke, G.,Witter, G., 1982. Häuser mit grünen pelz. Ein handbuch zur hausbegrünung.

[4] Bruse, M., Thönnessen, M. and Radtke, U., Practical and theoretical investigation of the influence of facade greening on the distribution of heavy metals in urban streets. Proceedings International Conference on Urban Climatology \& International congress of Biometeorology, Sydney, 8-12 (1999).

[5] Pekkanen, J., Timonen, K.L., Tiittanen, P., Vallius, M., Lanki, T., Sinkko, H., 2000. Exposure and Risk Assessment for Fine and Ultrafine Particles in Ambient Air. National Public Health Institute.

[6] Pope, A. C., Ezzati, M., Dockery, D.W., 2009. Fine-Particulate Air Pollution and Life Expectancy in the United States. The new England journal of medicine; N Engl J Med 2009; 360:376-86.

[7] Bartfelder, F., Köhler, M., 1987. Experimentelle untersuchungen zur function von fassadenbegrünungen, Berlin.

[8] Freer-Smith, P.H., Beckett, K.P., Taylor, G., 2004. Deposition velocities to Sorbus aria, Acer campestre, Populus deltoids x trichocarpa 'Beaupre', Pinus nigra and $x$ Cupressocyparis leylandii for coarse, fine and ultra-fine particles in the urban environment. Environmental Pollution 133 (2005) 157-167.

[9] Maher B, Moore C, Matzka J. Spatial variation in vehicle-derived metal pollution identified by magnetic and elemental analysis of road side tree leaves. Atmospheric Environment 2008:42: 364-373.

[10] Thönnessen, M., 2002. Elementdynamik in fassaden begrünendem wilden Wein (Parthenocissus tricuspidata), Kölner Geographischer Arbeiten, Heft 78, Köln. 
[11] Powe, N.A., Willis, K.G., Mortality and morbidity benefits of air pollution $\left(\mathrm{SO}_{2}\right.$ and $\left.\mathrm{PM}_{10}\right)$ adsorption attributable to woodland in Britain, Journal of Environmental Management , 70, 119-128 (2004).

[12] McCartney, H.A. et al., Electric charge and the deposition of spores of Barley Mildew Erysiphe Graminis, Atmospheric Environment, 16-5, 1133-1143 (1982).

[13] Nowak, D.J., Mcpherson, E.G., Rowntree, R.A., Chicago's Urban Forest Ecosystem: Results of the Chicago Urban Forest Climate Project, United States Department of Agriculture, General Technical Report NE-186, 1994

[14] http://www.epa.gov/air/airpollutants.html.

[15] Koch, K., Ensikat, H.J. The hydrophobic coatings of plant surfaces: Epicuticular wax crystals and their morphologies, crystallinity and molecular self-assembly. Micron, 39-7, 759-772 (2008).

[16] Niemietz, A., Wandelt, K., Barthlott, W. and Koch, K. Thermal evaporation of multi-component waxes and thermally activated formation of nanotubules for superhydrophobic surfaces. Progress in Organic Coatings, 66-3, 221-227 (2009).

[17] Samuels, L., Kunst, L. and Jetter, R. Sealing plant surfaces: cuticular wax formation by epidermal cells. Ann. Rev. Plant Biol., 59, 683-707 (2008).

[18] Müller, F., Michel, W., Schlicht, V., Tietze, A. and Winter, P. SelfCleaning surfaces using the Lotus effect. Handbook for Cleaning/Decontamination of Surfaces, 791-811 (2007).

[19] Mariq, M. in Nanoparticles in Medicine and Environment, Marijnissen, J. \& Gràdon, L., 19-37 (2010).

[20] Becquerel, A.C., Eléments de physique terrestre et de météorologie (1847)

[21] Becquerel, A.C., Des forces physico-chimiques et de leur intervention dans la production des phénomènes naturels (1875). 